\title{
CONVERGENCE FIELDS OF ROW-FINITE AND ROW-INFINITE REVERSIBLE MATRICES
}

\author{
ALBERT WILANSKY
}

In a recent paper (Proceedings of the American Mathematical Society vol. 1 (1950) pp. 397-401), P. Erdös and G. Piranian exposed a degree of arbitrariness in the relative size of the convergence fields of row-finite and row-infinite matrices. Roughly, they proved that there is a row-finite (-infinite) matrix with no equipotent rowinfinite (-finite) matrix.

This paper attempts to restore order. Also, remarks on reversible matrices are appended. A simplification in the above-mentioned paper will be described below, after Lemma 1 .

Summability is often investigated with a reversible matrix (see, e.g., Banach, Théorie des opérations linéaires, pp. 90-95) or the special case, called normal, of a triangular matrix with no zeros on the main diagonal, although $\mathrm{K}$. Zeller has recently weakened the requirements (Math. Zeit. vol. 53).

THEOREM. Given an arbitrary reversible matrix $A$, there is a matrix, all of whose rows are of infinite length, consistent with $A$, whose convergence field is identical with that of $A$. There is also a normal, conservative matrix whose convergence field includes that of $A$.

We conjecture that the first part of the theorem is true with finite instead of infinite.

The second part of the theorem may be compared with the following result which was proved, but not stated, by Erdös and Piranian.

There is a regular matrix whose convergence field is not contained in that of any normal matrix.

For their treatment of case I, p. 398, does not use regularity of $B$.

The proof of theorem is given in two lemmas.

LEMma 1. If, for a matrix $A$, there is a sequence $\left\{t_{n}\right\}, t_{n} \neq 0$, such that lim $_{n} t_{n} x_{n}=0$ for all $x$ which is summable $A$, then there is a matrix $B$, all of whose rows are of infinite length, consistent with $A$, whose convergence field is identical with that of $A$. There is also a normal, conservative matrix which sums all the sequences which are summable $A$.

The second part of the lemma is trivial. We set $d_{n n}=t_{n}, d_{n k}=0$ if $k \neq n$. Here $D$ turns out to be co-null. (See my paper, Trans. Amer. 1951.

Presented to the Society, December 28, 1951; received by the editors October 26, 
Math. Soc. vol. 67 (1949) p. 62, line 11.)

To prove the first part, suppose $\left\{t_{n}\right\}$ exists. We can clearly find a sequence $\left\{s_{n}\right\}, s_{n}>0$, such that $\sum s_{n} x_{n}$ converges for all $x$ which is summable $A$.

Let $b_{n k}=a_{n k}$ if either $a_{n m} \neq 0$ for some $m \geqq k$, or $k \leqq n, b_{n k}=s_{k}$ otherwise.

Then

$$
\sum_{k=1}^{\infty} b_{n k} x_{k}=\sum_{k=1}^{\infty} a_{n k} x_{k}+\sum_{k=r(n)}^{\infty} s_{k} x_{k}
$$

where $r(n) \geqq n$. Here $r(n)$ describes the length of the $n$th row of $A$ provided that that row is at least $n$ terms long, otherwise $r(n)=n$. For any $n$ for which $r(n)=\infty$, the last sum will be zero.

If $x$ is summable $A$, it is obviously summable $B$ to the same limit. If $x$ is summable $B$, the last series must converge so that the $B$-transform of $x$ will exist. Thus $x$ is summable $A$ to the same limit.

REMARK. Using this argument we can simplify the matrix given by Erdös and Piranian, p. 400 , by simply omitting every alternate row.

The following lemma shows that a single upper bound can be put on the growth of a sequence which is summable by a reversible matrix. Obviously the hypothesis of reversibility cannot be dropped, even for regular matrices.

Lемма 2. Let $A$ be a reversible matrix. Then there is a sequence $\left\{t_{n}\right\}$, $t_{n}>0$, such that $\lim t_{n} x_{n}=0$ for all $x$ which is summable $A$.

For example, if $A$ is the matrix of the Césaro-Hölder transform, $t_{n}=1 / n$ would do.

There is (Banach, loc. cit. p. 94) a sequence $\left\{\alpha_{i}\right\}$ and matrix $\left(c_{n k}\right)$ with

$$
x_{n}=\alpha_{n} \lim y_{i}+\sum_{k} c_{n k} y_{k}
$$

where $y_{n}=\sum_{k} a_{n k} x_{k}$; moreover $\sum_{k}\left|c_{n k}\right|<\infty$ for each $n$.

We choose $t_{n}>0$ such that $\lim _{n} t_{n} \sum_{k}\left|c_{n k}\right|=\lim _{n} t_{n} \alpha_{n}=0$. Then, for $x$ summable $A, y$ as above, we have $t_{n} x_{n}=t_{n} \alpha_{n} \lim y_{i}+t_{n} \sum_{k} c_{n k} y_{k}$, and this clearly proves the lemma, noting that $\left\{y_{n}\right\}$ is convergent, and therefore bounded.

The proof of the theorem is now complete.

We now append some remarks on reversible matrices. Let $A$ be reversible and $\left\{\alpha_{i}\right\},\left(c_{n k}\right)$ have the same meaning as in the proof of Lemma 2. 
Lemma 3. The matrix $C$ is a right inverse for $A$.

We set, for each $p, y=\delta^{p}$ in (1), where $\delta^{p}$ is the sequence of zeros, save for a 1 in the $p$ th place. This gives $x_{n}=c_{n p}$ and so $\sum_{k} a_{n k} c_{n \rho}$ $=\sum_{k} a_{n k} x_{k}=\delta_{n p}$. This proves the lemma.

LEMмA 4. If $\sum_{k} \sum_{r} a_{n k} c_{k r}=\sum_{r} \sum_{k} a_{n k} c_{k r}$ for each $n$, then $\alpha_{k}=0$ for all $k$. In particular, if $A$ is row-finite, $\alpha_{k}=0$ for all $k$.

We set $y_{k}=1$ in (1). This gives $x_{n}=\alpha_{n}+\sum_{k} c_{n k}$. Thus $1=\sum_{k} a_{n k} x_{k}$ $=\sum_{k} a_{n k} \alpha_{k}+\sum_{k} \sum_{r} a_{n k} c_{k r}=\sum_{k} a_{n k} \alpha_{k}+1$ by hypothesis and Lemma 3. Since $A$ is reversible, it follows that $\alpha_{k} \equiv 0$.

We finally give an example of a regular matrix which has a two-sided inverse but is not reversible.

Let $A$ be the matrix

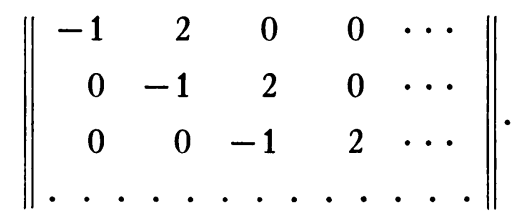

It has the two-sided inverse

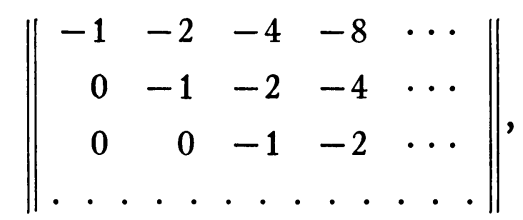

and yet is not reversible since it transforms the sequence $\left\{(1 / 2)^{n}\right\}$ to zero.

Thus, for a matrix, reversibility is not a generalization of the property of having an inverse.

LEHIGH UNIVERSITY 